\title{
Identification and control of sources of Taenia solium infection - the attempts to eradicate the parasite
}

\author{
Małgorzata Samorek-Pieróg, Jacek Karamon, Tomasz Cencek \\ Department of Parasitology and Invasive Diseases, \\ National Veterinary Research Institute, 24-100 Pulawy, Poland \\ j.karamon@piwet.pulawy.pl
}

Received: January 19, 2018

Accepted: March 15, 2018

\begin{abstract}
Taenia solium is a parasite causing porcine cysticercosis and human taeniosis and cysticercosis, parasitic zoonoses with a serious public health and economic influence. It has been globally ranked as the top foodborne parasite by the Food and Agriculture Organisation of the United Nations (FAO) and the World Health Organisation (WHO). This parasite is transmitted mainly in countryside regions where animals are free roaming, having access to human faeces, and infected pork is widely available. More developed countries eliminated cysticercosis; nonetheless, there are insufficient data about the current endemicity status of $T$. solium, due to increased human migration from endemic areas. Formally submitted statistics on cysticercosis in pigs are extremely inadequate. This is the result of not reporting all cases of the disease by some countries and lack of molecular verification during identification of the parasite. There is a need to develop diagnostic tests with increased sensitivity and specificity. The purpose of the present review is to summarise current knowledge about diagnostic and control methods concerning $T$. solium infection. The article does not address the diagnostics of human cysticercosis, since there is a distinct medical field which should be discussed separately. The paper focuses mainly on identifying the sources of $T$. solium infection, presenting the methods to detect and control porcine cysticercosis and taeniosis in humans.
\end{abstract}

Keywords: Taenia solium, cysticercosis, taeniosis, diagnostics, control.

\section{Introduction}

Taenia solium, solitary tapeworm of humans (pork tapeworm), is the causative agent of porcine cysticercosis and human taeniosis and cysticercosis. T. solium infection is considered a parasitic zoonosis with significant public health and economic consequences. It affects mostly less developed countries with particular cultural, socio-economic, and sanitary conditions, as Asia, sub-Saharan Africa, and Latin America (33). Nevertheless, a growing number of infections are revealed in Europe and the United States, mainly because of increased human migration from endemic regions (13). It has been globally ranked as the top foodborne parasite by the Food and Agriculture Organisation (FAO) of the United Nations and the World Health Organisation (WHO) in 2014 (11). $T$. solium also decreases the market value of pork and makes it hazardous to consume. The WHO Foodborne Disease Burden Epidemiology Reference Group recognised this parasite as the main reason of decease due to food-borne diseases, leading to an overall of 2.8 million disability-adjusted life-years (DALYs), in 2015 (45).

$T$. solium is registered by the WHO as a neglected tropical disease (NTD). The schedule determining strategic goals for control and elimination of the NTDs lists T. solium taeniasis/cysticercosis as requiring legalised strategies for eradication and control to be intensified by 2020 (43). In 2014, the WHO in partnership with the FAO, World Organisation for Animal Health (OIE), and International Livestock Research Institute (ILRI) held a meeting devoted to increased inspection of diseases caused by $T$. solium infections, taeniasis, and neurocysticercosis. A network led by the WHO was established to assist countries in 
their actions and to indicate national gaps in control. As a result, control programmes are being established in several countries. They need easy-to-use and affordable diagnostics to set a baseline to measure the effects of interventions and to conduct systematic supervision and proper diagnosis of clinical cases (44).

\section{Biology and transmission of Taenia solium}

Taenia solium has a complex life cycle, during which humans are the exclusive definitive hosts (Fig. 1). They harbour the mature form of the tapeworm, causing taeniasis. Both pigs and, occasionally, humans can be intermediate hosts, harbouring the larvae (cysticercus cellulose). Taeniasis appears in humans as a result of consumption of raw or undercooked pork meat contaminated with larvae (15). The cysticerci evaginate in the small intestine and the scolex adheres to the mucosa with its double row of hooks and its four suckers, begins forming proglottids and develops into the mature form. The strobila of a mature worm consists of several hundreds of proglottids, measuring 2-4 m. After two months from infection, gravid proglottids are expelled in the excrement and thus become the origin of infection for the intermediate host (each detached segment contains 50,000-60,000 fertile eggs) (34). When pig ingests faeces comprising $T$. solium eggs, larvae accommodate in the body of the animal, mainly in the subcutaneous fat, muscles, or brain. At the time of 10-12 weeks after infection, larvae transform into cysts. The infection is seldom related with any symptoms, because pigs are slaughtered before cysts achieve the degenerative stage, responsible for manifestations in humans (15).

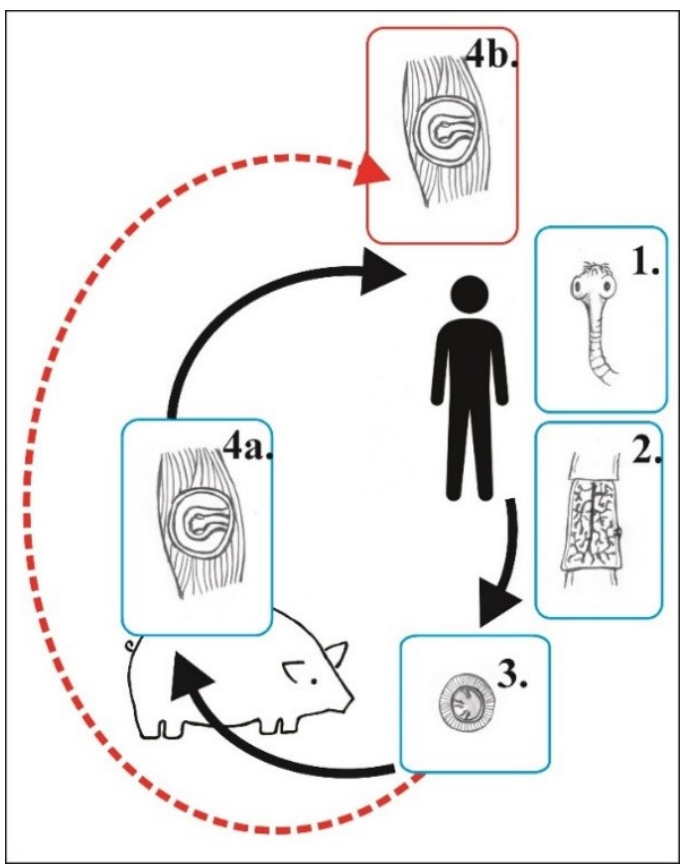

Fig. 1. Life cycle of Taenia solium. 1. - scolex; 2. - gravid proglottid, 3. - egg, 4a. - cysticercus in pig, 4b. cysticercus in human
Taeniasis is linked with gentle manifestations or none at all, nevertheless, identification of infection is really important due to the hazard of cysticercosis in the carrier or in the direct surroundings (15). The situation is diametrically different when human becomes the intermediate host. Then, the infection leads to cysticercosis or neurocysticercosis, much more dangerous in consequences. This can occur by drinking water or eating food defiled with parasite's eggs or by transferring eggs to mouth with dirty hands (especially in children). It has also been suggested that humans may be able to autoinfect themselves (external/internal autoinfection) $(21,34)$.

There are about 20 million infections with T. solium cysticerci in people and it is estimated that there are 50,000 demises every year due to neurocysticercosis (5). Symptoms, their intensity, and effects of this disease in humans depend on the individual sensitivity of the organism, the strength of infection, and location of larvae. Cysticercosis of muscles, even with intensive infection, may be asymptomatic. Nevertheless, when cysticerci are located in the brain, the condition is related with neurocysticercosis (NCC). It creates symptoms of the disease, like tumours or epilepsy, causing very serious health consequences, debilitation, and possibly leading to death (35). Most epilepsy cases around the globe are the effects of NCC; moreover, $30 \%$ of all cases of the disease occur in lands where T. solium is endemic (45).

It is obvious that there is an urgent need to improve methods of diagnosis and control of these zoonoses and, if possible, eradicate them. In spite of their considerable global impact, in terms of both economic burden and the number of infections, the data for precise estimation of distribution and transmission are limited. Nevertheless, the awareness of cysticercosis is increasing and bodies such as the WHO have put forward improvement strategies for the inspection of cysticercosis and taeniasis $(21,45)$.

\section{Diagnostics of taeniasis}

The efficacious detection of infections with $T$. solium in humans is extremely important because the parasite eggs excreted with human faeces are directly responsible for cysticercosis in people and, through cysticercosis in pigs, indirectly responsible for taeniasis in humans.

Microscopy was the first available method of diagnosis of taeniasis until the early 1990s. Nonetheless, two problems hinder proper identification of $T$. solium infections: insufficient sensibility of faeces microscopy and structural resemblance of the eggs of $T$. solium to the eggs of T. saginata. The presence of the double row of hooks on the scolex of $T$. solium enables to distinguish these two species. Furthermore, the structure of the gravid uterus in the distal proglottids and that of the genitalia in the mature 
proglottids provide precise species identification. However, it is really technically difficult, due to the fact that these proglottids are mostly unavailable in investigated materials $(15,34)$.

A coproantigen ELISA (copro-Ag-ELISA; coproantigen enzyme-linked immunosorbent assay) is one of the most accessible and efficacious diagnostic tools for intestinal taeniasis. It is used in epidemiological studies with sensitivity of $95 \%$ and specificity of about $99 \%$. This assay detects taenia-specific molecules in faecal samples, indicating tapeworm infection (1). Copro-AgELISA is predominantly genus-, not species-, specific and as a consequence cross-reacts with $T$. saginata. Hence, this test is not able to recognise precisely T. solium carriers. Notwithstanding, a species specificity of $100 \%$ revealed a hybrid test joining polyclonal antibodies versus Taenia adult tapeworm somatic extracts and an enzyme-conjugated rabbit IgG versus $T$. solium mature excretory-secretory antigen $(22,44)$. These tests are characterised by the following features: after a week of treatment coproantigens are undetectable, detection of coproantigens is likely earlier to patency and quantity is unrelated with the existence or amount of eggs. Furthermore, coproantigens are stable in not fixed stool samples stored at room temperature for days, in frozen samples, or in samples prepared with formalin stored at room temperature. They are stable for a long time, i.e. months to years (2).

Serological diagnosis of an adult intestinal Taenia solium infection can be reached by enzyme-linked immunoelectrotransfer blot method (EITB). This serological test provides diagnosis of species-specific circulating antibodies, and there are no cross-reactions with another parasitical infection. This provides an opportunity to conquer some of the troubles that persist with coproantigen examination (2). Specificity of this method was considered to be $100 \%$ and sensitivity about $95 \%$ (14). The principle of this test is to detect antibodies directed versus excretion-secretion (ES) antigens from unripe $T$. solium tapeworms that were bred in hamsters with immunosuppression. The ES antigens were described and two recombinant antigens of $33 \mathrm{kDa}(\mathrm{rES} 33)$ and $38 \mathrm{kDa}(\mathrm{rES} 38)$ were produced as substitute for native proteins. A disadvantage of this method can be the presence of antibodies in the organism, even though a patient might no longer have the parasite (8).

The currently available diagnostic methods for species-specific detecting taeniasis are unsatisfactory; thus copro-PCR assays have been developed as a result. Mayta et al. (32) developed a nested PCR where the sequence searched for was the gene sequence encoding the $T$. solium oncosphere-specific protein Tso31. The assay has $100 \%$ specificity and sensitivity under the field conditions and can be used for early detection and prophylaxis of taeniasis or cysticercosis. Multiplex PCR by Yamasaki et al. (47) was developed for differential identification of cysticercosis and taeniosis, together with their causative factors ( $T$. solium, $T$. saginata, or $T$. asiatica). Target sequence in this method encodes cytochrome $c$ oxidase subunit 1 gene yielded products specific for $T$. saginata, $T$. asiatica, and T. solium (with molecular sizes of 827, 269, 720, and $984 \mathrm{bp}$, respectively). Praet et al. (36) developed a real-time PCR where an 86-fragment inside the Internal Transcribed Spacer 1 (ITS1) sequence should be amplified and detected for T. solium and a 79-fragment within the ITS1 sequence should be amplified and detected for $T$. saginata. The assay has $99 \%$ specificity and $83 \%$ sensitivity, so it requires further studies to optimise the method. Restriction fragment length polymorphism (RFLP) PCR developed by Gonzalez et al. (20) enables differential identification of T. solium and $T$. saginata taeniosis/cysticercosis from different geographical positions, where the occurrence of the two parasites overlaps. In this assay amplification products received by the alternative PCRs protocols were digested by 6-base and 4-base recognising endonucleases (20). All these assays proved to be useful for differentiation of faecal eggs of $T$. solium, $T$. saginata, and $T$. asiatica (34). Importantly, the significance of the loop-mediate PCR assay for the diagnosis of taeniosis vectors is still increasing; however, it is necessary to optimise this method in the future (44).

\section{Diagnostics of porcine cysticercosis}

The effective detection of porcine cysticercosis is really important as consumption of infected pork is a direct cause of taeniasis in humans.

Diagnostics of porcine cysticercosis can be performed ante mortem (tongue and muscle inspection and serological detection of antibodies and antigen) and also post mortem (carcass inspection and molecular methods). These techniques enable to identify infected pigs and eliminate them from food chain. In this article we are focused on diagnostic methods available for identification of porcine cysticercosis and indicate which of them can be applied in humans.

The main diagnostic procedure for identification of metacestodes of $T$. solium in pig's carcass is meat inspection in slaughterhouse. This inspection of predilected sites (tongue, masticatory muscles, and heart) is obligatory due to Regulation (EC) No $854 / 2004$. Porcine cysticercosis can be also detected by tongue inspection or full/partial carcass dissection. Tongue inspection or palpation is a simple and specific test. It is a useful tool for rapid assessment of hot spots. Unfortunately, it has low sensitivity - only fewer than $30 \%$ of pigs with low intensity of infection can be identified. Full carcass dissection is a gold standard technique in diagnostics of cysticercosis in pigs. Nevertheless, it is not feasible in the daily practice because it is extremely laborious and expensive as the entire carcass must be sliced in more or less $0.5 \mathrm{~cm}$ 
cuts. The occurrence of cysticerci in muscles cannot be overlooked, because they are the source of infection in humans. The meat inspection in slaughterhouse is a simple and specific method, but with low sensitivity generally underestimating the status of cysticercosis in pig population. Cysts are readily visible by six weeks. After reaching mature form, they are generally oval, about the size of $10 \times 5 \mathrm{~mm}$, or bigger, with a transparent, white parasite membrane and host capsule. Pale fluid and the scolex, evident as a white dot, are visible inside the cyst (34).

Porcine cysticercosis can develop after infection with $T$. hydatigena, $T$. solium, and $T$. asiatica and sometimes differentiation between one another can be confusing, therefore researchers developed some confirmatory tests, like PCR, immunochemistry, and serological methods (Ag-ELISA, Ab-ELISA, LLGPEITB). Immunodiagnostic techniques applied in pigs have some advantages, i.e. greater sensitivity than carcass dissection, diagnosis on live animals, comparative affordability and simplicity to perform on numerous serum samples (33).

Ag-ELISA (antigen enzyme-linked immunosorbent assay) is used for the qualitative identification of viable larvae of Taenia spp. in porcine and human serum samples, and thus it is not intended for detection of calcified or degenerated larvae. The monoclonal antibodies (IgG isotype) applied in this test are designed versus excretory-secretory products (ESP) of viable $T$. saginata larvae. These antibodies detect the glycoprotein antigens prevalent on the tegument and in the ESP of metacestodes (10). In order to identify parasite antigens in serum, cerebrospinal fluid or urine, using polyclonal or monoclonal antibodies (B158/B60 Ag-ELISA, HP10 Ag-ELISA), a number of tests have been developed. What is more, in contrast to detection of antibodies, survey of circulating antigen amount enables to recognise the cases of cysticercosis with viable parasites, with antigen amount corresponding to the numbers and range of lesions. The test is genusspecific, not species-specific, so it is not possible to distinguish infections of separate Taenia species in pigs (T. solium, T. hydatigena, T. asiatica). It can be implemented to support ante mortem identification of porcine cysticercosis (aid meat inspectors and farmers to make the right decision) or serological controlling of anthelmintic treatment in pigs and humans (a rapid decrease in the level of antigens after effective antiparasitic therapy) $(8,10,44)$.

A specific antibody response reflects an infection with $T$. solium in human and pig organisms. Antibodydetecting techniques are unhelpful with monitoring changes over short periods, because they do not differentiate active infections from inactive ones. Nevertheless, they have much better diagnostic effectiveness than tests detecting antigens (3). Antibodies can be determined in serum, cerebrospinal fluid, tears, or saliva. Detection of $T$. solium specific antibodies in serum merely shows exposure to the parasite and does not necessarily establish the infection. What is more, it has been revealed that antibodies can be prevalent in the organism some time after removal of the parasite by anthelmintic treatment or immune response (8). A number of methods identify antibodies to $T$. solium infections in humans and pigs, like immunoblotting or ELISA. At the beginning, antigens applied in antibody-detection tests were ESP, cyst fluid, or raw homogenates from $T$. solium, $T$. saginata, or $T$. crassiceps larvae (8).

Ab-ELISA is a simple and sensitive method and allows for processing of numerous samples at the same time. Unfortunately, a number of agents influence its diagnostic ability: the specificity and sensitivity of particular method, the laboriousness of determining proper reference serum kit, and the application of raw or minimally purified antigens, causing non-specific reactions primarily with fasciolasis, angiostrongyliasis, schistosomiasis, echinococcosis, and sparganosis (37). Research on antigenic features of surface proteins and cyst fluid and revised techniques of purification have resulted in the elaboration of improved serological applications. Nowadays, many scientists tend to use synthetic or recombinant proteins $(7,38)$. It was shown that the specificity of these antigens is higher than that of native antigens; however, their sensitivity is predominantly lower (9).

EITB is currently considered the most specific test, with sensitivity of $98 \%$ and specificity of $100 \%$ (while examined in humans) (42). An improved fraction of glycoproteins (GP), received by purifying a raw larvae extract by chromatography with lentillectin (LLGP; lentil lectin purified glycoprotein), is applied. From an entire cysticercus homogenate, seven antigenic GPs, i.e. GP13, GP14, GP18, GP21, GP24, GP39-42, and GP50 (the numbers refer to molecular mass in kilodaltons) were isolated. The appearance of even a single antibody band determines a positive result. It is a commercially available assay and it has been extensively used for the diagnosis of human and porcine cysticercosis $(8,37,42)$.

PCR based tests with oligonucleotide primers received from species-specific DNA samples have a great potential in the differential identification of Taenia sp., offering really fast and sensitive methods for the identification of parasites in general (20). Primarily, PCR tests have been applied to identify doubtful T. solium cystic lesions in pork meat and also to confirm the results obtained during inspection of meat from slaughtered pigs (41). Karamon et al. (27) optimised three different PCR assays developed previously for identification of $T$. solium $(32,41,47)$. Comparison of the effectiveness of these PCRs revealed that the most sensitive and specific one is nested PCR by Mayta et al. (32). Moreover, two other methods used by Sreedevi et al. (41) based on techniques characterised initially by Yamasaki et al. (47) and Jardin et al. (25) have unsatisfactory specificity as unspecific products (T. saginata and E. granulosus, or 
only $T$. saginata) were also present. Hence these methods were considered of limited usefulness for identification of $T$. solium. This study encourages verification of the usability of some PCR methods and the choice and optimisation of the effectiveness of molecular tools for this purpose (27).

\section{Control of taeniasis and cysticercosis}

Taenia solium is one of the main causative agents of epileptic seizures and the highest classified foodborne parasite in respect of illness encumbrance around the world. The International Task Force for Disease Eradication has announced that this parasite was eradicated in 1993. Nevertheless, due to the restricted data about its spread, absence of sensitive treatment and identification methods, and the absence of implemented intervention packages it still persists as a neglected disease. At present there are no large scale control programmes validated against this parasite. However, currently available intervention instruments are very effective, which is very promising (46). The applied intervention tools include treatment of pigs, pig vaccination, mass administration of antiparasitic drugs to humans, health education, and simultaneous treatment of people and pigs. Nevertheless, control remains unsustainable and unsuccessful (6).

In order to eliminate established Taenia solium infections from pigs and thus minimise economic losses at slaughterhouses, pig producers treat animals with cysticidal drugs as anthelmintic treatment. Several antiparasitic drugs have been tested, e.g. albendazole sulphoxide, albendazole, praziquantel, oxfendazole, and flubendazole. The administration of oxfendazole turned out the most favourable approach. This benzimidazole drug is inexpensive, easy to administer, and efficacious against larval and adult gastrointestinal helminths. A single dose of $30 \mathrm{mg} / \mathrm{kg}$ (per os) kills all cysts in pig's muscles and after 17 days post-treatment the meat is considered as suitable for human consumption. Nevertheless, the cystic lesions are healed until three to six months after treatment. What is more, such treatment makes pigs resistant to subsequent infections for a long time after drug intake. Additional studies are required to check the safety of oxfendazole in piglets and pregnant sows $(12,17,19$, 35). Oxfendazole treatment, which reduces the number of infected pigs and also diminishes the probability of occurrence of taeniosis in humans, is a very promising approach for controlling transmission of $T$. solium to pigs. The main problem encountered in this method is that the majority of animals are free from cysticercus cellulosae (even in hyperendemic areas). Despite oxfendazole treatment, these animals are still vulnerable to new $T$. solium infections. To solve this problem antiparasitic treatment should be applied directly before pig slaughtering, and this is the time when any cyst after medicament intake could become the source of infection for humans (30). Another drawback of oxfendazole treatment is its limited affordability. Last but not least, oxfendazole is unavailable and not registered for application in pigs in the majority of developing countries so far (12).

Vaccines are yet another tool for control and prophylaxis of $T$. solium infections. Several vaccines have been used with positive preventive results against $T$. solium cysticercosis. Nevertheless, their efficacy has not been evaluated in natural environment, but only under controlled experimental conditions (40). Pig vaccination requires administration of several doses of a vaccine. This increases the expenses and hinders feasibility of the task. What is more, immunisation should be performed in very young piglets; it reduces the occurrence of existent infections, but does not eliminate larval forms of T. solium living in the piglet's organism at the time of vaccination. The perfect vaccine should have specific features, i.e. the ability to stop the life cycle of the parasite, affordability, accessibility, safety, and ability to diminish the spread of the disease to people and pigs $(15,30)$. Three vaccines: TSOL18, TSOL45, and S3Pvac have the biggest potential in practical application.

S3Pvac is a peptide-based synthetic vaccine. It consists of three peptides: KETc12, KETc1, and GK1, which are prevalent in all phases of $T$. solium development. This vaccine was successfully tested in the field in Mexico (23). Data from these studies revealed a decrease in intensity of infection by about $98 \%$ and a decrease in prevalence by about $50 \%$ in free-ranging piglets. The effects of these studies indicate the efficiency of the vaccination in endemic areas. Nonetheless, considering the period before and after the vaccination, there were no noticeable modifications in the local levels of transmission. Therefore, this examination revealed the restricted utility of one-fold vaccination as the only way to stop T. solium transmission in an endemic area $(23,40)$.

TSOL45 is a recombinant protein, experimentally tested in Mexico, which induced 97\% protection against $T$. solium (31). This protein is expressed solely on the surface of $T$. solium oncosphere (it is not present in adult tapeworms or cysticerci), meaning that this is stage-specific antigen. This protein elicits high-level protection against cysticercosis. Further investigations are needed due to the fact that a genetic variation of the TSOL45 gene has been noted between Mexican and Chinese $T$. solium, potentially affecting the efficiency of the vaccine (31).

TSOL18, similarly to TSOL45, is expressed on the surface of the oncosphere of the tapeworm. It is evidenced to be the most efficient vaccine versus T. solium transmission. Data from independent pilot vaccine attempts conducted in Cameroon, Honduras, Peru, and Mexico revealed $99.5 \%-100 \%$ protection in experimentally infected pigs $(4,18,29)$. Two attempts with the use of TSOL45 in the natural environment have been made. The first one was carried out in 
Cameroon by Assana et al. (4), where three-month piglets were administered three vaccinations along with a single dose of oxfendazole. Simultaneously, identical oxfendazole treatment was applied in control unvaccinated group. When pigs reached the age of 12 months the autopsy was conducted. It was observed that in the control group a percentage of animals with $T$. solium cysts was at the level of $19.6 \%$, whereas the vaccinated animals were devoid of any cysts. The vaccine had completely eliminated the spread of $T$. solium in the animals participating in the investigation $(4,29)$. In the second trial, conducted in Peru, TSOL18 was combined with another protective antigen - TSOL16, but without any anthelmintic treatment (26). This vaccine also appeared to be highly effective. The amount of viable cysts in the vaccinated animals was remarkably reduced compared with the control group.

Administration of anthelminthics to people can eliminate the source of infection caused by $T$. solium and thus contribute to the reduction of cysticercosis in pigs and humans. Treatment of taeniasis can be based on a diagnosis-treatment scheme or achieved by mass drug administration (MDA) (12). The most common taenicides are niclosamide (NCZ) and praziquantel (PZQ). The effectiveness the first one is $85 \%$ after a single dose. Due to a high price of treatment by NCZ, its use is now restricted in comparison to PZQ. It is a very efficient and safe medicine with efficacy around $95 \%$ (35). The effects of MDA to people in the form of a single intervention proved to be ineffective. Annual MDA yields better results, however, further studies are needed (29). However, the potential disadvantage of MDA is a threat of massive contamination of the environment with Taenia eggs during mass treatment of people. Neither PZQ nor NCZ affect the viability of taeniid eggs. In case of improper disposure and securement of excrements, it could potentially induce growth in human and porcine cysticercosis (29).

The attempts to use a combination of taenicide treatment in humans as well as in pig population with anthelmintic drug against $T$. solium revealed statistically significant decrease in the prevalence of both taeniosis in people and cysticercosis in pigs and people. The results were evaluated over a 20-month period and yet elimination was not attained (16). The limited information regarding sustainability of single MDA implies that after the end of treatment, transmission will very quickly return to the previous level due to the fact that a small number of tapeworm carriers is able to preserve transmission (28).

Health education can be considered the main agent in developing an efficient and sustainable intervention strategy to reduce $T$. solium transmission. It necessitates multidisciplinary contribution (involving the collaboration between veterinary, medical, social, and environmental sectors) and cooperation with the society. Due to the fact that it is a rather long term intervention tool, requiring broad social commitment, this strategy can be expensive. However, teaching about the parasite and the consequences of zoonoses in endemic areas translates, with time, into behavioural and practical changes necessary to reduce the occurrence of the diseases. These changes include: personal hygiene (washing hands after defecation), improved sanitation (use of latrines), eating habits (hygienic preparation of dishes), improving pig husbandry system (corralling pigs, pig treatment and vaccination), and improving veterinary sanitary measures (meat inspection). People involved in health education have to incessantly increase and update their knowledge to be well prepared to achieve this task (12, 39). Education programmes should be initiated together with prevention and control programmes. Because these interventions may not be economically feasible for many developing countries, they should be supported by such government policies as purchasing infected meat at market prices, provisioning a subsidy to farmers who breed healthy animals for slaughter (certified meat, or providing some form of compensation for carcass condemnation). Otherwise, farmers may try to evade veterinary control and turn to unsupervised abattoirs or practice home slaughter (33).

In conclusion, Taenia solium is mainly transmitted in less developed countries in countryside regions, where pigs are free roaming, having access to human faeces, and infected pork is widely available. Improvement of sanitation and pig husbandry system eliminated cysticercosis in more developed countries. Nonetheless, there is little information about current endemicity status of $T$. solium, because of increased human migration from endemic areas where strategies for eradication and control are urgently required. Official notification on porcine cysticercosis is extremely inadequate due to the fact that most cases are diagnosed based only on morphology, without molecular verification, and moreover not all countries report their findings. There is a need to develop diagnostic tests with increased sensitivity and specificity in order to distinguish active cysticercosis from its inactive form. What is more, affordable and practical diagnostic tests should be constructed to encourage their application in developing countries.

Currently, no intervention has persistently interrupted the transmission that is so urgently needed. A possible strategy to eradicate $T$. solium would comprise a combination of approaches concentrating on pigs (husbandry system, treatment and vaccination) and humans (treatment and health education). The prosperity of any intervention tool is mostly determined by the level of political and social acceptance and commitment.

The prominent progression in development of diagnostic techniques, treatment, and prevention of transmission of Taenia solium has occurred in recent years. Many international scientists have been collaborating over the years, sharing ideas and resources concerning $T$. solium taeniasis/cysticercosis 
(13). A network of collaborating groups was created through the patronage of the World Health Organisation as the Cysticercosis Working Group in Peru and the Cysticercosis Working Group in Asia (24). These groups are working towards more effective identification, treatment, and eradication of cysticercosis. European scientists have extended their collaborations into a European Network on taeniosis/cysticercosis, CYSTINET, joining 22 European Union countries (www.cystinet.org). They are focused on the development of advanced, feasible diagnostic and control tools, estimations of disease burden and its economic impact, as well as unification of standardised reporting and administration procedures (13).

Conflict of Interests Statement: The authors declare that there is no conflict of interests regarding the publication of this article.

Financial Disclosure Statement: The review was financially supported by statutory activity of the National Veterinary Research Institute in Pulawy, Poland.

Animal Rights Statement: None required.

\section{References}

1. Allan J.C., Avila G., Garcia Noval J., Flisser A., Craig P.S.: Immunodiagnosis of taeniasis by coproantigen detection. Parasitology 1990, 101, 473-477.

2. Allan J.C., Wilkins P.P., Tsang V.C.W., Craig P.S.: Immunodiagnostic tools for taeniasis. Acta Trop 2003, 87, 87-93.

3. Assana E., Kanobana K., Tume C.B., Zoli P.A., Nguekam, Geerts S., Berkvens D., Dorny P.: Isolation of a $14 \mathrm{kDa}$ antigen from Taenia solium cyst fluid by HPLC and its evaluation in enzyme linked immunosorbent assay for diagnosis of porcine cysticercosis. Res Vet Sci 2007, 82, 370-376.

4. Assana E., Kyngdon C.T., Gauci C.G., Geerts S., Dorny P., De Deken R., Anderson G.A., Zoli A.P., Lightowlers M.W.: Elimination of Taenia solium transmission to pigs in a field trial of the TSOL18 vaccine in Cameroon. Int J Parasitol 2010, 40, 515-519.

5. Bern C., Garcia H.H., Evans C., Gonzalez A.E., Verastegui M., Tsang V.C., Gilman R.H.: Magnitude of the disease burden from neurocysticercosis in a developing country. Clin Infect Dis 1999, 29, 1203-1209.

6. Braae U.C., Devleesschauwer B., Gabriel S., Dorny P., Speybroeck N., Magnussen P., Torgerson P., Johansen M.V.: CystiSim - An agent-based model for Taenia solium transmission and control. PLoS Negl Trop Dis 2016, 10, e0005184.

7. Chung J.Y., Kang S.Y., Bahk Y.Y., Kong Y., Huh S., Cho S.Y.: A Recombinant 10-kDa Protein of Taenia solium metacestodes specific to active neurocysticercosis. J Infect Dis 1999, 180, 1307-1315.

8. Deckers N., Dorny P.: Immunodiagnosis of Taenia solium taeniosis/cysticercosis. Trends Parasitol 2010, 26, 137-144.

9. Dorny P., Brandt J., Zoli A., Geerts S.: Immunodiagnostic tools for human and porcine cysticercosis. Acta Tropica 2003, 87, $79-86$.
10. Dorny P., Phiri I.K., Vercruysse J., Gabriel S., Willingham A.L. $3^{\text {rd }}$, Brandt J., Victor B., Speybroeck N., Berkvens D.: A Bayesian approach for estimating values for prevalence and diagnostic test characteristics of porcine cysticercosis. Int J Parasitol 2004, 34, 569-576.

11. FAO/WHO: Multicriteria-based ranking for risk management of food-borne parasites. Report of a Joint FAO/WHO Expert Meeting 3-7 September 2012, FAO Headquarters, Rome, Italy. FAO/WHO, Geneva, 2014, p. 322.

12. Gabriël S., Dornya P., Mwape K.E., Trevisan C., Braae U.C., Magnussen P., Thys S., Bulaya C., Phiri I.K., Sikasunge C.S., Makungu C., Afonso S., Nicolau Q., Johansen M.V.: Control of Taenia solium taeniasis/cysticercosis: The best way forward for sub-Saharan Africa? Acta Trop 2017, 165, 252-260.

13. Gabriël S., Johansen M.V., Pozio E., Smit G.S., Devleesschauwer B., Allepuz A., Papadopoulos E., van der Giessen J., Dorny P.: Human migration and pig/pork import in the European Union: What are the implications for Taenia solium infections? Vet Parasitol 2015, 213, 38-45.

14. Garcia-Noval J., Allan J.C., Fletes C., Moreno E., DeMata F., Torres-Alvarez R., Soto de Alfaro H., Yurrita P., HiguerosMorales H., Mencos F., Craig P.S.: Epidemiology of Taenia solium taeniasis and cysticercosis in two rural Guatemalan communities. Am J Trop Med Hyg 1996, 55, 282-289.

15. Garcia H.H., Gonzalez A.E., Evans C.A., Gilman R.H.: Cysticercosis Working Group in Peru.: Taenia solium cysticercosis. Lancet 2003, 362, 547-556.

16. Garcia H.H., Gonzalez A.E., Gilman R.H., Moulton L.H., Verastegui M., Rodriguez S., Gavidia C., Tsang V.C.: Combined human and porcine mass chemotherapy for the control of T. solium. Am J Trop Med Hyg 2006, 74, 850-855.

17. Gonzalez A.E., García H.H., Gilman R.H., Tsang V.C.: Control of Taenia solium. Acta Trop 2003, 87, 103-109.

18. Gonzalez A.E., Gauci C.G., Barber D., Gilman R.H., Tsang V.C., Garcia H.H., Verastegui M., Lightowlers M.W.: Vaccination of pigs to control human neurocysticercosis. Am J Trop Med Hyg 2005, 72, 837-839.

19. Gonzalez A.E., Gavidia C., Falcon N., Bernal T., Verastegui M., Garcia H.H., Gilman R.H., Tsang V.C.: Protection of pigs with cysticercosis from further infections after treatment with oxfendazole. Am J Trop Med Hyg 2001, 65, 15-28.

20. González L.M., Montero E., Puente S., López-Velez R., Hernández M., Sciutto E., Harrison L.J., Parkhouse R.M., Gárate T.: PCR tools for the differential diagnosis of Taenia saginata and Taenia solium taeniasis/cysticercosis from different geographical locations. Diagn Microbiol Infect Dis 2002 42, 243-249.

21. Gripper L.B., Welburn S.C.: Neurocysticercosis infection and disease - A review. Acta Trop 2017, 166, 218-224.

22. Guezala M.C., Rodriguez S., Zamora H., Garcia H.H., Gonzalez A.E., Tembo A., Allan J.C., Craig P.S.: Development of a speciesspecific coproantigen ELISA for human Taenia soliumtaeniasis. Am J Trop Med Hyg 2009, 81, 433-437.

23. Huerta M., de Aluja A.S., Fragoso G., Toledo A., Villalobos N., Hernández M., Gevorkian G., Acero G., Díaz A., Alvarez I., Avila R., Beltrán C., Garcia G., Martinez J.J., Larralde C., Sciutto E.: Synthetic peptide vaccine against Taenia solium pig cysticercosis: successful vaccination in a controlled field trial in rural Mexico. Vaccine 2001, 12, 262-266.

24. Ito A., Wandra T., Yamasaki H., Nakao M., Sako Y., Nakaya K., Margono S.S., Suroso T., Gauci C., Lightowlers M.W.: Cysticercosis/taeniasis in Asia and the Pacific. Vector Borne Zoonotic Dis 2004, 4, 95-107.

25. Jardim E.A.G.V., Linhares G.F.C., Torres F.A.G., Araujo J.L.B., Barbosa S.M.: Specific discrimination between Taenia saginata and Taenia solium by one step PCR assay and duplex-PCR. Ciencia Rural, Santa Maria 2006, 36, 166-172.

26. Jayashi C.M., Kyngdon C.T., Gauci C.G., Gonzalez A.E., Lightowlers M.W.: Successful immunization of naturally reared pigs against porcine cysticercosis with a recombinant oncosphere antigen vaccine. Vet Parasitol 2012, 188, 261-267. 
27. Karamon J., Sroka J., Cencek T., Różycki M., Chmurzyńska E., Bilska-Zając E., Zdybel J., Nowak P., Kędzierska J., Dębiak P.: Optimisation and comparison of three PCR procedures for molecular identification of Taenia solium. Bull Vet Inst Pulawy 2013, 57, 507-512.

28. Kyvsgaard N.C., Johansen M.V., Carabin H.: Simulating transmission and control of Taenia solium infections using a Reed-Frost stochastic model. Int J Parasitol 2007, 37, 547-558.

29. Lightowlers M.W.: Control of Taenia solium taeniasis/ cysticercosis: past practices and new possibilities. Parasitology 2013, 140, 1566-1577.

30. Lightowlers M.W.: Eradication of Taenia solium cysticercosis: A role for vaccination of pigs. Int J Parasitol 2010, 15, 1183 1192.

31. Martinez-Ocaña J., Romero-Valdovinos M., de Kaminsky R.G., Maravilla P., Flisser A.: Immunolocalization of TSOL18 and TSOL45-1A, the successful protective peptides against porcine cysticercosis, in Taenia solium oncospheres. Parasit Vectors 2011, 4:3.

32. Mayta H., Gilman R.H., Prendergast E., Castillo J.P., Tinoco Y.O., Garcia H.H., Gonzalez A.E., Sterling C.R., Cysticercosis Working Group in Peru.: Nested PCR for specific diagnosis of Taenia solium taeniasis. J Clin Microbiol 2008, 46, 286-289.

33. Murrel K.D., Dorny P., Flisser A., Geerts S., Kyvsgaard N.C., McManus D., Nash T., Pawlowski Z.:WHO/FAO/OIE Guidelines for the surveillance, prevention and control of taeniosis/cysticercosis. OIE, WHO, FAO, Geneva, 2005.

34. OIE: Manual of Diagnostic Tests and Vaccines for Terrestrial Animals. Paris 2008, chapter 2.9.5.

35. Pawlowski Z., Allan J., Sarti E.: Control of Taenia solium taeniasis/cysticercosis: From research towards implementation. Int J Parasitol 2005, 35 1221-1232.

36. Praet N., Verweij J.J., Mwape K.E., Phiri I.K., Muma J.B., Zulu G., van Lieshout L., Rodriguez-Hidalgo R., Benitez-Ortiz W., Dorny P., Gabriel S.: Bayesian modelling to estimate the test characteristics of coprology, coproantigen ELISA and a novel real-time PCR for the diagnosis of taeniasis. Trop Med Int Health 2013, 18, 608-614.

37. Rodriguez S., Wilkins P., Dorny P.: Immunological and molecular diagnosis of cysticercosis. Pathog Glob Health 2012, 106, 286-298
38. Sako Y., Nakao M., Ikejima T., Piao X.Z., Nakaya K., Ito A.: Molecular characterization and diagnostic value of Taenia solium low-molecular-weight antigen genes. J Clin Microbiol 2000, 38, 4439-4444.

39. Sarti E., Rajshekhar V.: Measures for the prevention and control of Taenia solium taeniosis and cysticercosis. Acta Trop 2003, 87, 137-143.

40. Sciutto E., Morales J., Martinez J.J., Toledo A., Villalobos M.N., Cruz-Revilla C., Meneses G., Hernandez M., Diaz A., Rodarte L.F., Acero G., Gevorkian G., Manoutcharian K., Paniagua J., Fragoso G., Fleury A., Larralde R., De Aluja A.S., Larralde C.: Further evaluation of the synthetic peptide vaccine S3Pvac against Taenia solium cysticercosis in pigs in an endemic town of Mexico. Parasitology 2007, 134, 129-133.

41. Sreedevi C., Hafeez M., Kumar P.A., Rayulu V.C., Subramanyam K.V., Sudhakar K.: PCR test for detecting Taenia solium cysticercosis in pig carcasses. Trop Anim Health Prod 2012, 44, 95-99.

42. Tsang V.C., Brand J.A., Boyer A.E.: An enzyme-linked immunoelectrotransfer blot assay and glycoprotein antigens for diagnosing human cysticercosis (Taenia solium). J Infect Dis 1989, 159, 50-59.

43. WHO: Accelerating work to overcome the global impact of neglected tropical diseases - A roadmap for implementation. WHO, Geneva, 2012, p. 42.

44. WHO: Taenia solium Taeniasis/Cysticercosis Diagnostic Tools. Report of a Stakeholder Meeting, 17-18 December 2015. WHO, Geneva, 2015, p. 26.

45. WHO:Taeniasis/cysticercosis (Updated March 2017) URL: http://www.who.int/mediacentre/factsheets/fs376/en/WHO, 2017.

46. WHO: Working to overcome the global impact of neglected tropical diseases. First WHO report on neglected tropical diseases. WHO, Geneva, 2010, p. 184.

47. Yamasaki H., Allan J.C., Sato M.O., Nakao M., Sako Y., Nakaya K., Qiu D., Mamuti W., Craig P.S., Ito A.: DNA Differential diagnosis of taeniasis and cysticercosis by multiplex PCR. J Clin Microbiol 2004, 42, 548-553. 